UFIFT-HEP-97-17

RU97-32

May 1997

\title{
A Model of Yukawa Hierarchies
}

\author{
John K. Elwood, Nikolaos Irges and Pierre Ramond" \\ Institute for Fundamental Theory, \\ Department of Physics, University of Florida \\ Gainesville FL 32611, USA
}

\begin{abstract}
We present a model for the observed hierarchies among the Yukawa couplings of the standard model in the context of an effective low energy theory with an anomalous $U(1)$ symmetry. This symmetry, a generic feature of superstring compactification, is a remnant of the Green-Schwarz anomaly cancellation mechanism. The gauge group is that of the standard model, augmented by $X$, the anomalous $U(1)$, and two family-dependent phase symmetries $Y^{(1)}$ and $Y^{(2)}$. The correct hierarchies are reproduced only when $\sin ^{2} \theta_{w}=3 / 8$ at the cut-off. To cancel anomalies, right-handed neutrinos and other standard model singlets must be introduced. Independently of the charges of the right-handed neutrinos, this model produces the same neutrino mixing matrix and an inverted hierarchy of neutrino masses. The heaviest is the electron neutrino with a mass $\sim 1 \mathrm{meV}$, and mixing of the order of $\lambda_{c}^{3}$ with each of the other two neutrinos.
\end{abstract}

\footnotetext{
${ }^{1}$ Supported in part by the United States Department of Energy under grants DE-FG0586-ER40272 and (for PR) DE-FG02-96ER-40559
} 


\section{Introduction}

Theories of extended objects, superstring theories in particular, offer the best hope for resolving the difference between General Relativity and Quantum Mechanics. When compactified to four dimensions, these models naturally reproduce Yang-Mills interactions of the type found in Nature. Unfortunately, this conceptual matching between the "fundamental" and the "observed" has not yet resulted in a detailed picture, capable of relating the parameters of the standard model. One reason, the disparity of scales, which are $\leq \mathrm{TeV}$ for the standard model, but $\leq 10^{19} \mathrm{GeV}$ for superstring theories, can be obviated by employing low energy supersymmetry. This allows for a perturbative journey of the standard model almost to the Planck scale. There, a more integrated structure seems to emerge: the unification of the three gauge couplings, and the subject of this paper, the appearance of some order among the Yukawa couplings, are both evident. This order among the Yukawa couplings can most easily be described as an expansion in the Cabibbo angle $\lambda_{c}$, where we find a geometric interfamily hierarchy

$$
\begin{array}{rlrl}
\frac{m_{u}}{m_{t}} & =\mathcal{O}\left(\lambda_{c}^{8}\right) ; & & \frac{m_{c}}{m_{t}}=\mathcal{O}\left(\lambda_{c}^{4}\right) ; \\
\frac{m_{d}}{m_{b}}=\mathcal{O}\left(\lambda_{c}^{4}\right) ; & \frac{m_{s}}{m_{b}}=\mathcal{O}\left(\lambda_{c}^{2}\right), \\
\frac{m_{e}}{m_{\tau}}=\mathcal{O}\left(\lambda_{c}^{4}\right) ; & \frac{m_{\mu}}{m_{\tau}}=\mathcal{O}\left(\lambda_{c}^{2}\right),
\end{array}
$$

among fermions of the same charge. There is also an intrafamily hierarchy

$$
\frac{m_{b}}{m_{t}}=\mathcal{O}\left(\lambda_{c}^{3}\right), \quad \frac{m_{b}}{m_{\tau}}=\mathcal{O}(1) .
$$

Finally, the CKM quark mixing matrix is of the form [4]

$$
\mathcal{U}_{C K M} \sim\left(\begin{array}{ccc}
1 & \lambda_{c} & \lambda_{c}^{3} \\
\lambda_{c} & 1 & \lambda_{c}^{2} \\
\lambda_{c}^{3} & \lambda_{c}^{2} & 1
\end{array}\right),
$$

where $\sim$ indicates only order of magnitude estimates. Although expressed in terms of fermion mass ratios, these correspond to relations among Yukawa couplings. The one exception is $m_{b} / m_{t}$, which depends on the angle $\beta$ that links the vacuum values of the two Higgs in the minimal supersymmetric model. It is therefore possible to discuss the origin of these exponents in the context of both exact supersymmetry and electroweak symmetries. Below we present a simple model that reproduces these hierarchies. 


\section{Effective Low Energy Theories with Green- Schwarz Anomalies}

A wide class of superstring theories compactified to four dimensions 2 yield $N=1$ supersymmetric effective low energy theories with cut-off $M_{\text {string }} \leq$ $M_{\text {Planck }}$, and a universal gauge coupling $g_{\text {string. Their gauge structure includes }}$ a visible sector containing at least the standard model gauge groups and several gauged phase symmetries $Y^{(1)}, Y^{(2)}, \ldots$. In addition, they contain hidden gauge interactions with unknown gauge structure. These two sectors are linked by several gauged Abelian symmetries, one of which, denoted by $X$, is anomalous. A subset of these Abelian symmetries are broken below the cut-off by a stringy mechanism [3] that generates a Fayet-Iliopoulos term in the $D$-term of the $X$ symmetry

$$
\xi^{2}=-\frac{g_{\text {string }}^{3}}{192 \pi^{2}} M_{\text {Planck }}^{2} C_{\text {grav }},
$$

where $C_{\text {grav }}$ is the mixed gravitational anomaly of the $X$ current

$$
C_{\text {grav }}=(X T T),
$$

the brackets stand for the sum over the particles in the triangle graph, and $T$ is the energy momentum tensor. For the remainder of this work, we shall denote the cutoff scale by $M$. In our convention, $C_{\text {grav }}$ is negative. Since its breaking occurs below the cut-off, it is legitimate to include $X$ as a symmetry of the low energy theory. We will also require that this vacuum preserve supersymmetry, since its scale is near the Planck mass.

The anomalies of the $X$-symmetry are compensated at the cut-off by a dimension five term in the effective Lagrangian

$$
\mathcal{L}=\frac{1}{g_{\text {string }}^{2}} \sum_{j} k_{j} F_{\mu \nu}^{[j]} F_{\mu \nu}^{[j]}+i \frac{\eta}{M_{\text {string }}} \sum_{j} k_{j} F_{\mu \nu}^{[j]} \tilde{F}_{\mu \nu}^{[j]}+\mathcal{L}_{\text {matter }}+\cdots,
$$

where the sum is over the gauge groups, and the $k_{j}$ are the Kac-Moody levels. under the $X$ gauge transformation, the axion-like field $\eta$ shifts as a NambuGoldstone boson, accounting for the anomalies in the $X$ current

$$
\partial_{\mu} j_{\mu}^{X} \sim \sum_{j} C_{j} F_{\mu \nu}^{j} \widetilde{F}_{\mu \nu}^{j}
$$

as long as the ratio $C_{j} / k_{j}$ is universal. This is the four-dimensional equivalent of the Green-Schwarz anomaly cancellation mechanism [⿰亻弋 all other anomaly coefficients to vanish. $C_{\text {color }}, C_{\text {weak }}$ and $C_{Y}$ are the mixed anomalies between the $X$ current and the standard model gauge currents,

$$
\left(X G^{A} G^{B}\right)=\delta^{A B} C_{\text {color }} ; \quad\left(X W^{a} W^{b}\right)=\delta^{a b} C_{\text {weak }} ; \quad(X Y Y)=C_{Y},
$$


where $G^{A}$ are the QCD currents, and $W^{a}$ the weak isospin currents. We must have

$$
\frac{C_{\text {grav }}}{12}=\frac{C_{\text {color }}}{k_{\text {color }}}=\frac{C_{\text {weak }}}{k_{\text {weak }}}=\frac{C_{\mathrm{Y}}}{k_{\mathrm{Y}}} \neq 0,
$$

and

$$
\left(X Y^{(i)} Y^{(j)}\right)=\delta^{i j} C^{(i)} .
$$

All the other anomaly coefficients must vanish by themselves

$$
\left(Y^{(i)} Y^{(j)} Y^{(k)}\right)=\left(Y^{(i)} Y^{(j)} Y\right)=\left(Y^{(i)} G^{A} G^{B}\right)=\left(Y^{(i)} W^{a} W^{b}\right)=\left(Y^{(i)} Y Y\right)=0 .
$$

as well as

$$
\left(X Y Y^{(i)}\right)=(X X Y)=\left(X X Y^{(i)}\right)=\left(Y^{(i)} T T\right)=0 .
$$

In theories with $N$ symmetries, the number of conditions to be satisfied increases as $N^{3}$, while the number of matter fields is limited by asymptotic freedom; it is therefore reasonable to expect that all charges could be uniquely determined by anomaly cancellations.

A consequence of this mechanism is that the Weinberg angle at cut-off can be understood [5] as a ratio of anomaly coefficients

$$
\tan ^{2} \theta_{w}=\frac{g_{Y}^{2}}{g_{\text {weak }}^{2}}=\frac{k_{\text {weak }}}{k_{Y}}=\frac{C_{\text {weak }}}{C_{Y}} .
$$

These anomaly coefficients can be computed from the $X$-charges of chiral fermions. Such fermions can come in two varieties, those from the three chiral families and those from standard model pairs with chiral $X$ values. The anomaly coefficients from the three chiral families can be related to the $X$ charges of the standard model invariants. The minimal supersymmetric standard model contains the invariants

$$
\mathbf{Q}_{i} \overline{\mathbf{u}}_{j} H_{u} ; \quad \mathbf{Q}_{i} \overline{\mathbf{d}}_{j} H_{d} ; \quad L_{i} \bar{e}_{j} H_{d} ; \quad H_{u} H_{d}
$$

where $i, j$ are the family indices, with $X$ charges

$$
X_{i j}^{[u]}, \quad X_{i j}^{[d]}, \quad X_{i j}^{[e]}, \quad X^{[\mu]},
$$

respectively; a simple computation yields

$$
\begin{aligned}
C_{\text {color }} & =\sum_{i}^{3}\left(X_{i i}^{[u]}+X_{i i}^{[d]}-3 X^{[\mu]}\right), \\
C_{Y}+C_{\text {weak }}-\frac{8}{3} C_{\text {color }} & =2 \sum_{i}^{3}\left(X_{i i}^{[e]}-X_{i i}^{[d]}+2 X^{[\mu]}\right) .
\end{aligned}
$$

Since the Kac-Moody levels of the non-Abelian factors are the same, the GreenSchwarz condition requires

$$
C_{\text {weak }}=C_{\text {color }}
$$


from which we deduce

$$
C_{Y}=\sum_{i}^{3}\left(\frac{5}{3} X_{i i}^{[u]}-\frac{1}{3} X_{i i}^{[d]}+2 X_{i i}^{[e]}-3 X^{[\mu]}\right) .
$$

Similar equations hold for the mixed anomalies of the $Y^{(i)}$ currents; their vanishing imposes constraints on the $Y^{(i)}$ charges of the standard model invariants.

The further constraint that the Weinberg angle be at its canonical $S U(5)$ value, $\sin ^{2} \theta_{w}=3 / 8$, that is $3 C_{Y}=5 C_{\text {weak }}$, yields the relations

$$
\begin{gathered}
X^{[\mu]}=\sum_{i}^{3}\left(X_{i i}^{[d]}-X_{i i}^{[e]}\right) . \\
C_{\text {color }}=\sum_{i}\left[X_{i i}^{[u]}-2 X_{i i}^{[d]}+3 X_{i i}^{[e]}\right],
\end{gathered}
$$

as well as

$$
C_{\text {color }}=\frac{1}{2} \sum_{i \neq j}\left[X_{i j}^{[u]}-2 X_{i j}^{[d]}+3 X_{i j}^{[e]}\right] .
$$

Since $C_{\text {color }}$ does not vanish, these equations imply that some standard model invariants have non-zero $X$ charges. In the framework of an effective field theory, it means that these invariants will appear in the superpotential multiplied by fields that balance the excess $X$ charge. These higher dimension interactions are suppressed by inverse powers of the cut-off [6]; this is the origin of Yukawa hierarchies and mixings.

A theory with extra Abelian gauged symmetries $X, Y^{(1)}, \ldots, Y^{(N)}$ will contain $N+1$ standard model singlet chiral superfields $\theta_{1}, \ldots \theta_{N+1}$, to serve as their order parameters. The anomaly-induced supersymmetry-preserving vacuum is determined by the vanishing of the $N+1 D$ terms

$$
\begin{aligned}
\sum_{a=1}^{N+1} x_{a}\left|\theta_{a}\right|^{2} & =\xi^{2}, \\
\sum_{a=1}^{N+1} y_{a}^{(k)}\left|\theta_{a}\right|^{2} & =0, \quad k=1,2, \ldots, N .
\end{aligned}
$$

These equations can be solved as long as the $(N+1) \times(N+1)$ matrix $\mathrm{A}$, with rows equal to the $N+1$ vectors $\mathbf{x}=\left(x_{1}, x_{2}, \ldots, x_{N+1}\right), \mathbf{y}^{(k)}=\left(y_{1}^{(k)}, y_{2}^{(k)}, \ldots, y_{(N+1)}^{(k)}\right)$ has an inverse with a positive first row.

A typical term in the superpotential, invariant under these $N+1$ symmetries will then be of the form

$$
\mathbf{Q}_{i} \overline{\mathbf{d}}_{j} H_{d} \prod_{a}\left(\frac{\theta_{a}}{M}\right)^{n_{i j}^{[a]}}
$$


where holomorphy requires the $n_{i j}^{[a]}$ to be zero or positive integers. Invariance under the $N+1$ symmetries yields

$$
\begin{gathered}
X_{i j}^{[d]}+\sum_{a} x_{a} n_{i j}^{[a]}=0, \\
Y_{i j}^{(k)[d]}+\sum_{a} y_{a}^{(k)} n_{i j}^{[a]}=0, \quad k=1,2, \ldots, N .
\end{gathered}
$$

These involve the same matrix $A$, and here a solution also requires that $\operatorname{det} A \neq$ 0 , linking hierarchy to vacuum structure. Evaluated at the vacuum values of the $\theta_{a}$ fields, the terms shown above can produce a family-dependent Yukawa hierarchy.

A successful model of this type is highly constrained: it must satisfy all anomaly conditions and reproduce the observed Yukawa hierarchies. Additionally, the breaking triggered by the anomalous $U(1)_{X}$ must preserve supersymmetry, as well as the standard model gauge symmetries. In searching for models of this type, we assume that the $X$ charge is family-independent, and that the $Y^{(i)}$ charges are traceless over the families. In this way, the $Y^{(i)}$ are responsible for the interfamily hierarchy and mixing while the $X$ charges account for the intrafamily structure.

The role of the anomalous symmetry in generating hierarchies has been proposed earlier [7, 8, 9, 10, 11], but with a family-dependent $X$ symmetry. In previous works, it was pointed out how the Weinberg angle is related to the hierarchy [8, 9] and that the seesaw mechanism implies $R$-parity conservation 12]. Below we present a model in which all of these features are satisfied.

\section{The Model}

In this simple illustrative model, there are three gauged symmetries beyond the standard model: a family-independent anomalous $X$, and two family-traceless symmetries $Y^{(1)}, Y^{(2)}$. On the three chiral families, they are

$$
Y^{(1)}=(B+L)\left(\begin{array}{ccc}
2 & 0 & 0 \\
0 & -1 & 0 \\
0 & 0 & -1
\end{array}\right),
$$

where $B$ and $L$ are baryon and lepton numbers, and the diagonal matrix is in family space. The other charges are

$$
Y^{(2)}=\left(\begin{array}{ccc}
1 & 0 & 0 \\
0 & 0 & 0 \\
0 & 0 & -1
\end{array}\right),
$$

for $\mathbf{Q}, \overline{\mathbf{u}}, \bar{e}$ and zero for $L, \overline{\mathbf{d}}$. We assume that the only dimension-three term in the superpotential is the Yukawa coupling for the top quark,

$$
W=\mathbf{Q}_{3} \overline{\mathbf{u}}_{3} H_{u} \text {. }
$$


The family tracelessness of $Y^{(1)}$ and $Y^{(2)}$ insures the vanishing of the contribution of the the chiral families of many anomaly coefficients

$$
\left(Y^{(i)} G^{A} G^{B}\right)_{f}=\left(Y^{(i)} W^{a} W^{b}\right)_{f}=\left(Y^{(i)} Y Y\right)_{f}=\left(Y^{(i)} T T\right)=\left(X Y Y_{i}\right)_{f}=0 .
$$

The model assumes no fermions with standard model quantum numbers except for those in the MSSM. It therefore follows from the above equations that the Higgs pair is vector-like with respect to the $Y^{(1,2)}$ charges. Since $\left(X Y Y^{(1,2)}\right)$ must vanish over the Higgs pair, we infer that their charges are also vector-like with respect to $X$. Hence all the charges of the $\mu$ term vanish

$$
X^{[\mu]}=Y^{(1)[\mu]}=Y^{(2)[\mu]}=0,
$$

which is also favored by the independent vacuum analysis [12]. The other anomaly conditions involving the hypercharge must be satisfied by the chiral fermions

$$
\left(Y^{(1)} Y^{(1)} Y\right)_{f}=\left(Y^{(2)} Y^{(2)} Y\right)_{f}=\left(Y^{(1)} Y^{(2)} Y\right)_{f}=0 .
$$

For these to hold, it is not sufficient to invoke family-tracelessness, but our assignment clearly satisfies these equations. Other anomaly conditions that do not involve standard model currents are computed to be

$$
\begin{aligned}
& \left(Y^{(1)} Y^{(1)} Y^{(1)}\right)_{f}=\left(Y^{(1)} Y^{(1)} Y^{(2)}\right)_{f}=6, \\
& \left(Y^{(2)} Y^{(2)} Y^{(2)}\right)_{f}=\left(Y^{(1)} Y^{(2)} Y^{(2)}\right)_{f}=0 .
\end{aligned}
$$

These anomalies need to be canceled by other fields, some of which must be the $\theta$ fields whose vacuum values break the $X$ and $Y^{(1,2)}$ symmetries. These do not suffice to saturate the anomaly conditions with rational charge assignments, however. More fields must be added; some will be interpreted as the righthanded partners of the standard model neutrinos.

The charges of the $\theta$ fields are constrained by the observed Yukawa hierarches, which are reproduced by

$$
A^{-1}=\left(\begin{array}{ccc}
1 & 0 & 0 \\
1 & 0 & -1 \\
1 & 1 & -1
\end{array}\right),
$$

so that all three $\theta$ fields ave the same vacuum expectation value

$$
\left|<\theta_{1}>\right|=\left|<\theta_{2}>\right|=\left|<\theta_{3}>\right|=\xi .
$$

Their charges are given by

$$
A=\left(\begin{array}{ccc}
1 & 0 & 0 \\
0 & -1 & 1 \\
1 & -1 & 0
\end{array}\right),
$$


and the $\theta$ sector contributions to the anomalies are

$$
\begin{gathered}
\left(Y^{(1,2)} T T\right)_{\theta}=\left(Y^{(1)} Y^{(1)} Y^{(1)}\right)_{\theta}=\left(Y^{(2)} Y^{(2)} Y^{(2)}\right)_{\theta}=0 \\
\left(Y^{(1)} Y^{(1)} Y^{(2)}\right)_{\theta}=\left(Y^{(1)} Y^{(2)} Y^{(2)}\right)_{\theta}=-1 .
\end{gathered}
$$

Clearly more fields must be added, and from hereon, our construction is somewhat arbitrary, guided mostly by anomaly cancellation with rational charges. We introduce three $S O(10)$-like right-handed neutrinos with $Y^{(1,2)}$ charges of the same family structure as the chiral families:

\begin{tabular}{|c|c|c|c|}
\hline Charge & $\bar{N}_{1}$ & $\bar{N}_{2}$ & $\bar{N}_{3}$ \\
\hline \hline$X$ & $-1 / 2$ & $-1 / 2$ & $-1 / 2$ \\
\hline$Y^{(1)}$ & -2 & 1 & 1 \\
\hline$Y^{(2)}$ & -1 & 0 & 1 \\
\hline
\end{tabular}

which contribute to three anomaly coefficients

$$
\left(Y^{(1)} Y^{(1)} Y^{(1)}\right)_{\bar{N}}=-6 ; \quad\left(Y^{(1)} Y^{(1)} Y^{(2)}\right)_{\bar{N}}=-3 ; \quad\left(Y^{(1)} Y^{(2)} Y^{(2)}\right)_{\bar{N}}=-1 .
$$

Their $X$ charges insure, through the seesaw mechanism, $R$-parity conservation [12]. We also introduce four additional standard model singlets to cancel the remaining anomalies:

\begin{tabular}{|c|c|c|c|c|}
\hline Charge & $S_{1}$ & $S_{2}$ & $S_{3}$ & $S_{4}$ \\
\hline \hline$X$ & 1 & 0 & 0 & -1 \\
\hline$Y^{(1)}$ & $-3 / 2$ & $-1 / 2$ & $1 / 2$ & $3 / 2$ \\
\hline$Y^{(2)}$ & $1 / 2$ & $3 / 2$ & $-1 / 2$ & $-3 / 2$ \\
\hline
\end{tabular}


Their contributions to the anomalies are

$$
\begin{array}{cc}
\left(Y^{(1)} Y^{(1)} Y^{(1)}\right)_{S}=0 ; & \left(Y^{(1)} Y^{(1)} Y^{(2)}\right)_{S}=-2 \\
\left(Y^{(1)} Y^{(2)} Y^{(2)}\right)_{S}=2 ; & \left(Y^{(2)} Y^{(2)} Y^{(2)}\right)_{S}=0 .
\end{array}
$$

Hence the sum of all the $\left(Y^{(i)} Y^{(j)} Y^{(k)}\right)$ coefficients vanish. It is worth pointing out that the structure of the $S$-field sector is not crucial to any of the predictions of this paper, and its inclusion serves only to demonstrate a particular mechanism for cancelling anomalies. In fact, one must generally take care not to unleash unwanted vacuum flat directions as more $S$ fields are included in the model.

Three of the five $X$ charges of the chiral families are determined from the conditions $(X X Y)_{f}=0, C_{\text {color }}=C_{\text {weak }}$, and $\sin ^{2} \theta_{w}=3 / 8$ at unification, yielding

$$
X_{\mathbf{Q}}=X_{\overline{\mathbf{u}}}=X_{\bar{e}} \equiv a ; \quad X_{L}=X_{\overline{\mathbf{d}}} \equiv d .
$$

Using the top quark Yukawa constraint and the neutrality of the $\mu$ term,we find

$$
C_{\text {color }}=C_{\text {weak }}=\frac{3}{5} C_{Y}=3 a+d
$$

We also find

$$
\left(X Y^{(1)} Y^{(2)}\right)_{f}=0
$$

Hence

$$
\left(X Y^{(1)} Y^{(2)}\right)=\left(X Y^{(1)} Y^{(2)}\right)_{\bar{N}}+\left(X Y^{(1)} Y^{(2)}\right)_{S}=0 .
$$

The remaining anomalies that do not vanish are all determined in terms of $a$ and $d$

$$
\begin{array}{r}
(X X X)=10 a^{3}+5 d^{3}+\frac{21}{8} ; \quad C_{\text {grav }}=10 a+5 d+\frac{3}{2} \\
\left(X Y^{(1)} Y^{(1)}\right)=12 a+14 d+\frac{5}{2} ; \quad\left(X Y^{(2)} Y^{(2)}\right)=20 a+\frac{5}{2}
\end{array}
$$

The expansion parameter is therefore fully determined in terms of the string coupling constant, the Planck mass and the $X$ charges of the chiral families.

\subsection{Quark Yukawa Hierarchies}

The family structure of the charge $2 / 3$ Yukawa couplings is determined by the $Y^{(1,2)}$ charges as well as by $A^{-1}$. The invariant Yukawa interaction in the superpotential is

$$
\mathbf{Q}_{i} \overline{\mathbf{u}}_{j} H_{u}\left(\frac{\theta_{1}}{M}\right)^{n_{i j}^{(1)}}\left(\frac{\theta_{2}}{M}\right)^{n_{i j}^{(2)}}\left(\frac{\theta_{3}}{M}\right)^{n_{i j}^{(3)}}
$$


Invariance under the three charges yields

$$
\begin{aligned}
& n_{i j}^{(1)}=0, \\
& n_{i j}^{(2)}=Y_{i j}^{(2)[u]}, \\
& n_{i j}^{(3)}=-Y_{i j}^{(1)[u]}+Y_{i j}^{(2)[u]},
\end{aligned}
$$

where we have used the fact that $X$ is family independent and that $X^{[u]}=0$ from the top quark mass. The exponents $n_{i j}^{(1)}, n_{i j}^{(2)}$ are easily computed; all are either zero or positive integers, so that there are no supersymmetric zeros. The orders of magnitude in the charge $2 / 3$ Yukawa matrix are therefore

$$
Y^{[u]} \sim\left(\begin{array}{ccc}
\lambda^{8} & \lambda^{5} & \lambda^{3} \\
\lambda^{7} & \lambda^{4} & \lambda^{2} \\
\lambda^{5} & \lambda^{2} & 1
\end{array}\right)
$$

where

$$
\lambda=\frac{|\theta|}{M} .
$$

This matrix reproduces the geometric interfamily hierarchy in this sector,

$$
\frac{m_{u}}{m_{t}} \sim \lambda_{c}^{8}, \quad \frac{m_{c}}{m_{t}} \sim \lambda_{c}^{4},
$$

and its left-handed diagonalization is of the CKM form

$$
\left(\begin{array}{ccc}
1 & \lambda & \lambda^{3} \\
\lambda & 1 & \lambda^{2} \\
\lambda^{3} & \lambda^{2} & 1
\end{array}\right)
$$

with the expansion parameter identified with the Cabibbo angle $\lambda_{c}$.

In the down sector, the corresponding exponents are given by

$$
\begin{aligned}
& p_{i j}^{(1)}=-X^{[d]}, \\
& p_{i j}^{(2)}=-X^{[d]}+Y_{i j}^{(2)[d]}, \\
& p_{i j}^{(3)}=-X^{[d]}-Y_{i j}^{(1)[d]}+Y_{i j}^{(2)[d]} .
\end{aligned}
$$

To avoid supersymmetric zeros in all matrix elements, $X^{[d]}$ must be a negative integer or zero. The $Y^{(1,2)}$ charges of the down matrix elements are

$$
\left(Y^{(1)[d]}, Y^{(2)[d]}\right)=\left(\begin{array}{rrr}
(0,-1) & (1,-1) & (1,-1) \\
(-1,-2) & (0,-2) & (0,-2) \\
(-1,-3) & (0,-3) & (0,-3)
\end{array}\right) .
$$

A supersymmetric zero can develop in the (33) position if $X^{[d]}>-3$. To reproduce the observed hierarchy we must therefore require

$$
X^{[d]} \leq-3 .
$$


With this proviso, the down Yukawa matrix orders of magnitude are

$$
Y^{[d]} \sim \lambda_{c}^{-3 X^{[d]}-6}\left(\begin{array}{ccc}
\lambda_{c}^{4} & \lambda_{c}^{3} & \lambda_{c}^{3} \\
\lambda_{c}^{3} & \lambda_{c}^{2} & \lambda_{c}^{2} \\
\lambda_{c} & 1 & 1
\end{array}\right)
$$

which leads to its left-diagonalization by a matrix with the CKM structure. Hence the CKM mixing matrix is reproduced, with the correct identification of the expansion parameter with the Cabibbo angle

$$
\mathcal{U}_{C K M} \sim\left(\begin{array}{ccc}
1 & \lambda_{c} & \lambda_{c}^{3} \\
\lambda_{c} & 1 & \lambda_{c}^{2} \\
\lambda_{c}^{3} & \lambda_{c}^{2} & 1
\end{array}\right)
$$

and the hierarchy

$$
\frac{m_{d}}{m_{b}} \sim \lambda_{c}^{4}, \quad \frac{m_{s}}{m_{b}} \sim \lambda_{c}^{2},
$$

All interfamily hierarchies are reproduced in the quark sectors. The intrafamily quark hierarchy is

$$
\frac{m_{b}}{m_{t}}=\cot \beta \lambda_{c}^{-3 X^{[d]}-6},
$$

implying a suppression determined by the value of the color anomaly.

\subsection{Charged Lepton Hierarchies}

The exponents of the charged lepton sector are given by

$$
\begin{aligned}
& q_{i j}^{(1)}=-X^{[e]}, \\
& q_{i j}^{(2)}=-X^{[e]}+Y_{i j}^{(2)[e]}, \\
& q_{i j}^{(3)}=-X^{[e]}-Y_{i j}^{(1)[e]}+Y_{i j}^{(2)[e]},
\end{aligned}
$$

indicating that $X^{[e]}$ must also be a negative integer or zero. This is consistent with the canonical value of the Weinberg angle, for which $X^{[e]}=X^{[d]}$. The $Y^{(1,2)}$ charges in the charged lepton Yukawa matrix are

$$
\left(Y^{(1)[e]}, Y^{(2)[e]}\right)=\left(\begin{array}{lll}
(0,-1) & (3,-2) & (3,-3) \\
(-3,-1) & (0,-2) & (0,-3) \\
(-3,-1) & (0,-2) & (0,-3)
\end{array}\right) .
$$

If $X^{[e]} \leq-6$ there are no supersymmetric zeros, and one can check that the observed $e-\mu-\tau$ hierachy is not reproduced. If $X^{[e]}=-5$, there is one supersymmetric zero in the (13) position, but again the hierarchy comes out 
wrong. It is only with $X^{[e]} \geq-4$, with at least two supersymmetric zeros in the (12) and (13) positions, that one reproduces the observed pattern with

$$
Y^{[e]} \sim \lambda_{c}^{-3 X^{[e]}-6}\left(\begin{array}{ccc}
\lambda_{c}^{4} & 0 & 0 \\
\lambda_{c}^{7} & \lambda_{c}^{2} & 1 \\
\lambda_{c}^{7} & \lambda_{c}^{2} & 1
\end{array}\right) .
$$

Thus the constraints

$$
-3 \geq X^{[d]}=X^{[e]} \geq-4,
$$

reproduce the lepton hierarchy

$$
\frac{m_{e}}{m_{\tau}} \sim \lambda_{c}^{4}, \quad \frac{m_{\mu}}{m_{\tau}} \sim \lambda_{c}^{2},
$$

with two solutions:

$$
\frac{m_{b}}{m_{\tau}} \sim 1 ; \quad \frac{m_{b}}{m_{t}} \sim \cot \beta \lambda_{c}^{3} \quad \text { or } \quad \cot \beta \lambda_{c}^{6} .
$$

The latter case is not viable as it implies that $\beta \sim 0$, but the first yields an acceptable mass ratio with $\tan \beta \sim 1$. In either case, this ratio is naturally suppressed. The left-diagonalization of this matrix yields half the lepton mixing matrix

$$
\left(\begin{array}{ccc}
1 & \lambda_{c}^{9} & \lambda_{c}^{11} \\
\lambda_{c}^{9} & 1 & 1 \\
\lambda_{c}^{11} & 1 & 1
\end{array}\right)
$$

indicating large mixing between the $\mu$ and the $\tau$, and no mixing with the electron.

\subsection{Neutrino Hierarchies and Mixing}

The coupling of the right-handed neutrinos to the standard model is of the form

$$
L_{i} \bar{N}_{j} H_{u}\left(\frac{\theta_{1}}{M}\right)^{r_{i j}^{(1)}}\left(\frac{\theta_{2}}{M}\right)^{r_{i j}^{(2)}}\left(\frac{\theta_{3}}{M}\right)^{r_{i j}^{(3)}}
$$

with the integer exponents given by

$$
\begin{aligned}
r_{i j}^{(1)} & =-X^{[\nu]} \\
r_{i j}^{(2)} & =-X^{[\nu]}+Y_{i j}^{(2)[\nu]}, \\
r_{i j}^{(3)} & =-X^{[\nu]}-Y_{i j}^{(1)[\nu]}+Y_{i j}^{(2)[\nu]},
\end{aligned}
$$

and

$$
X^{[\nu]}=d-2 a-\frac{1}{2},
$$


must be a negative integer or zero to avoid supersymmetric zeros everywhere. $Y^{(i)}[\nu]$ are the charges of the invariants $L_{i} \bar{N}_{j} H_{u}$ given by

$$
\left(Y^{(1)[\nu]}, Y^{(2)[\nu]}\right)=\left(\begin{array}{rrr}
(0,1) & (3,2) & (3,3) \\
(-3,1) & (0,2) & (0,3) \\
(-3,1) & (0,2) & (0,3)
\end{array}\right) .
$$

If $X^{[\nu]}=0$, there is a supersymmetric zero in the (12) entry. If $X^{[\nu]} \leq-1$ and integer, there are no supersymmetric zeros, and if $X^{[\nu]}$ is positive or fractional there are no couplings between the standard model and the $\bar{N}$.

First if $X^{[\nu]} \leq-1$, we have

$$
Y^{[\nu]} \sim \lambda_{c}^{-3 X^{[\nu]}}\left(\begin{array}{ccc}
\lambda_{c}^{2} & \lambda_{c} & \lambda_{c}^{3} \\
\lambda_{c}^{5} & \lambda_{c}^{4} & \lambda_{c}^{6} \\
\lambda_{c}^{5} & \lambda_{c}^{4} & \lambda_{c}^{6}
\end{array}\right) .
$$

On the other hand, if $X^{[\nu]}=0$, the same matrix reads

$$
Y^{[\nu]} \sim\left(\begin{array}{ccc}
\lambda_{c}^{2} & 0 & \lambda_{c}^{3} \\
\lambda_{c}^{5} & \lambda_{c}^{4} & \lambda_{c}^{6} \\
\lambda_{c}^{5} & \lambda_{c}^{4} & \lambda_{c}^{6}
\end{array}\right)
$$

Additionally, Majorana mass terms for the right-handed neutrinos,

$$
M \bar{N}_{i} \bar{N}_{j}\left(\frac{\theta_{1}}{M}\right)^{t_{i j}^{(1)}}\left(\frac{\theta_{2}}{M}\right)^{t_{i j}^{(2)}}\left(\frac{\theta_{3}}{M}\right)^{t_{i j}^{(3)}}
$$

are generally allowed, where the powers $t_{i j}^{(1,2,3)}$ are given by Eqs. (3.79-81) with $X^{[\nu]}=-\frac{1}{2}$. The charges of the $\bar{N}_{i} \bar{N}_{j}$ combinations are

$$
\left(Y^{(1)[0]}, Y^{(2)[0]}\right)=\left(\begin{array}{ccc}
(-4,-2) & (-1,-1) & (-1,0) \\
(-1,-1) & (2,0) & (2,1) \\
(-1,0) & (2,1) & (2,2)
\end{array}\right) .
$$

which implies supersymmetric zeros in the (11) and (22) positions, and the Majorana mass matrix

$$
Y^{[0]} \sim \lambda_{c}^{2}\left(\begin{array}{ccc}
0 & 1 & \lambda_{c}^{2} \\
1 & 0 & \lambda_{c} \\
\lambda_{c}^{2} & \lambda_{c} & \lambda_{c}^{3}
\end{array}\right)
$$

Diagonalization of this matrix yields the eigenvalues $\lambda_{c}^{2}, \lambda_{c}^{2}$, and $\lambda_{c}^{5}$, which describe, in the absence of electroweak breaking, one Dirac pair with mass $\sim M \lambda_{c}^{2}$ and one Majorana mass $\sim M \lambda_{c}^{5}$.

Electroweak breaking causes these states to mix with the neutrinos of the standard model, through the seesaw mechanism [13]. Two cases must be considered separately. 
When $X^{[\nu]} \leq-1$, the effective neutrino Yukawa mixing is given by

$$
\widehat{Y}^{[\nu]} \sim \frac{v_{u}^{2}}{M} \lambda_{c}^{-6 X^{[\nu]}}\left(\begin{array}{ccc}
1 & \lambda_{c}^{3} & \lambda_{c}^{3} \\
\lambda_{c}^{3} & \lambda_{c}^{6} & \lambda_{c}^{6} \\
\lambda_{c}^{3} & \lambda_{c}^{6} & \lambda_{c}^{6}
\end{array}\right) .
$$

This leads to the neutrino masses

$$
m_{\nu_{e}} \sim \frac{v_{u}^{2}}{M} \lambda_{c}^{-6 X^{[\nu]}} ; \quad m_{\nu_{\mu}} \sim m_{\nu_{\tau}} \sim \frac{v_{u}^{2}}{M} \lambda_{c}^{-6 X^{[\nu]}-6} .
$$

The MNS 14 neutrino mixing matrix works out to be

$$
\mathcal{U}_{M N S} \sim\left(\begin{array}{ccc}
1 & \lambda_{c}^{3} & \lambda_{c}^{3} \\
\lambda_{c}^{3} & 1 & 1 \\
\lambda_{c}^{3} & 1 & 1
\end{array}\right),
$$

which shows that the electron neutrino mixing angle with the others is of the order of $\lambda_{c}^{3}$ while the $\mu$ and $\tau$ neutrinos mix together with large angles. With $v_{u} \sim 250 \mathrm{GeV}$ and $M \sim 10^{17} \mathrm{GeV}$, the electron neutrino mass is at most

$$
m_{\nu_{e}} \sim \lambda_{c}^{6} \times(.6 \mathrm{meV}),
$$

when $X^{[\nu]}=-1$. This leads to a very small value, detectable only in vacuum oscillation of the solar neutrinos .

The other case, $X^{[\nu]}=0$, yields more massive neutrinos. In this case, we have a supersymmetric zero in the Dirac mass matrix, leading to

$$
\widehat{Y}^{[\nu]} \sim \frac{v_{u}^{2}}{M \lambda_{c}}\left(\begin{array}{ccc}
1 & \lambda_{c}^{3} & \lambda_{c}^{3} \\
\lambda_{c}^{3} & \lambda_{c}^{5} & \lambda_{c}^{5} \\
\lambda_{c}^{3} & \lambda_{c}^{5} & \lambda_{c}^{3}
\end{array}\right) .
$$

We obtain the same MNS lepton mixing matrix as in the previous case, produce the same inverted neutrino mass hierarchy, but obtain different orders of magnitude estimates for the neutrino masses

$$
m_{\nu_{e}} \sim \frac{v_{u}^{2}}{M \lambda_{c}} \sim 5 \times 10^{-3,-4} \mathrm{eV} ; \quad m_{\nu_{\mu}} \sim m_{\nu_{\tau}} \sim 10^{-6,-7} \mathrm{eV} .
$$

This mass range and mixing for the electron neutrino is consistent with the small angle MSW [15] solution to the solar neutrino crisis.

The most striking result of this analysis is the inverted hierarchy among neutrino masses. Different choices for the $\bar{N}$ charges do not change this hierarchy nor the nature of the mixing. The reason is that the order of magnitude matrices factorize, and therefore the $\bar{N}$ charge contributions to the exponents cancel in the seesaw matrix. Thus, in our model, this inverted hierarchy is a consequence of the charge assignment of the charged leptons!

One of us would like to acknowledge the hospitality of the Rutgers particle theory group where this paper was completed. 


\section{References}

[1] L. Wolfenstein, Phys. Rev. Lett. 51 (1983) 1945.

[2] T. Kobayashi and H. Nakano, hep-th/9612066, and references therein. See also A. Faraggi, Nucl. Phys. B387 (1992) 239, ibid. B403 (1993) 101, ibid. B407 (1993) 57.

[3] M. Dine, N. Seiberg and E. Witten, Nucl. Phys. B289 (1987) 317; J. Atick, L. Dixon and A. Sen, Nucl. Phys. B292 (1987) 109.

[4] M. Green and J. Schwarz, Phys. Lett. B149 (1984) 117.

[5] L. Ibáñez, Phys. Lett. B303 (1993) 55.

[6] C. Froggatt and H. B. Nielsen Nucl. Phys. B147 (1979) 277.

[7] L. Ibáñez and G. G. Ross, Phys. Lett. B332 (1994)100.

[8] P. Binétruy and P. Ramond, Phys. Lett. B350 (1995) 49.

[9] Y. Nir, Phys. Lett. B354 (1995) 107.

[10] V. Jain and R. Shrock, Phys. Lett. B352 (1995) 83.

[11] K. Choi, E. Chun, and H. Kim, hep-ph/9611293.

[12] P. Binétruy, N. Irges, S. Lavignac, and P. Ramond, UFIFT-HEP-96-25, (hep-ph/9610481), to be published in Physics Letters.

[13] M. Gell-Mann, P. Ramond, and R. Slansky in Sanibel Talk, CALT-68709, Feb 1979, and in Supergravity (North Holland, Amsterdam 1979). T. Yanagida, in Proceedings of the Workshop on Unified Theory and Baryon Number of the Universe, KEK, Japan, 1979.

[14] Z. Maki, M. Nakagawa,and S. Sakata, Prog. Theo. Phys. 28,247(1962).

[15] L. Wolfenstein, Phys Rev D17 (1978)2369; S. Mikheyev and A. Yu Smirnov, Nuovo Cim. 9C (1986)17.

B406 (1993) 19. 